Draft version March 26, 2022

Preprint typeset using $\mathrm{L}_{\mathrm{T}} \mathrm{EX}$ style emulateapj v. 08/22/09

\title{
MAGNETIC FIELDS FROM QCD PHASE TRANSITIONS
}

\author{
Alexander G. Tevzadze ${ }^{1}$, Leonard Kisslinger ${ }^{2}$, Axel BrandenburG ${ }^{3,4}$, And Tina Kahniashvili ${ }^{2,5,6}$ \\ ${ }^{1}$ Faculty of Exact and Natural Sciences, Javakhishvili Tbilisi State University, 1 Chavchavadze Ave., Tbilisi, 0128, Georgia \\ ${ }^{2}$ McWilliams Center for Cosmology and Department of Physics, Carnegie Mellon University, 5000 Forbes Ave, Pittsburgh, PA 15213 \\ ${ }^{3}$ Nordita, KTH Royal Institute of Technology and Stockholm University, Roslagstullsbacken 23, 10691 Stockholm, Sweden \\ ${ }^{4}$ Department of Astronomy, AlbaNova University Center, Stockholm University, 10691 Stockholm, Sweden \\ ${ }^{5}$ Department of Physics, Laurentian, University, Ramsey Lake Road, Sudbury, ON P3E 2C, Canada \\ ${ }^{6}$ Abastumani Astrophysical Observatory, Ilia State University, 3-5 Cholokashvili Str., Tbilisi, 0194, Georgia \\ (Dated:) \\ Draft version March 26, 2022
}

\begin{abstract}
We study the evolution of QCD phase transition-generated magnetic fields in freely decaying MHD turbulence of the expanding Universe. We consider a magnetic field generation model that starts from basic non-perturbative QCD theory and predicts stochastic magnetic fields with an amplitude of the order of $0.02 \mu \mathrm{G}$ and small magnetic helicity. We employ direct numerical simulations to model the MHD turbulence decay and identify two different regimes: "weakly helical" turbulence regime, when magnetic helicity increases during decay, and "fully helical" turbulence, when maximal magnetic helicity is reached and an inverse cascade develops. The results of our analysis show that in the most optimistic scenario the magnetic correlation length in the comoving frame can reach $10 \mathrm{kpc}$ with the amplitude of the effective magnetic field being $0.007 \mathrm{nG}$. We demonstrate that the considered model of magneto-genesis can provide the seed magnetic field for galaxies and clusters.

Subject headings: primordial magnetic fields; early Universe
\end{abstract}

\section{INTRODUCTION}

The origin of the observed magnetic fields (MFs) in galaxies and clusters of $\sim 10^{-6}-10^{-5}$ Gauss (G) remains a matter of debate (Beck et al. 1996; Widrow 2002; Vallée 2004). Recently several different groups (Neronov \& Vovk 2010; Tavecchio et al.|2010; Dolag et al. 2011; Taylor et al 2011; Huan et al. 2011) reported the detection of a lower bound on a largescale correlated MF amplitude of the order of $10^{-16}$ $10^{-15} \mathrm{G}$, or possibly two orders of magnitude smaller (Dermer et al. 2011; Takahashi et al. 2012) at Mpc scales through blazar observations. One of the possible explanations of the large-scale correlated MF assumes the presence of a seed primordial magnetic field (PMF) which was generated during or prior to the radiation dominated epoch. This MF should satisfy several conditions: (i) The PMF should preserve approximate spatial isotropy, it has to be weak enough when its energy density can be treated as a first order of perturbation; (ii) The PMF should be smaller than the MF in galaxies by a few orders of magnitude at least, since during structure formation PMFs get amplified; (iii) Since the PMF energy density $\rho_{B}$ contributes to the radiation field, the big bang nucleosynthesis (BBN) bound implies $\Omega_{B} h_{0}^{2}=\rho_{B} / \rho_{\mathrm{cr}} \leq 2.4 \times 10^{-6}$ Grasso \& Rubinstein 2001), where $\rho_{\mathrm{cr}}$ is the critical density, ${ }^{1}$ and $h_{0}$ is the Hubble constant in units of $100 \mathrm{~km} \mathrm{~s}^{-1} \mathrm{Mpc}^{-1}$.

The possible origin of the PMF from the two major cosmological phase transitions, the electroweak phase transition (EWPT) and the QCD phase transition (QCDPT) (see Grasso \& Rubinstein 2001; Widrow 2002;

\footnotetext{
${ }^{1}$ The ratio of $\rho_{B}$ to the energy density of the radiation $\rho_{\text {rad }}$ is constant during cosmological evolution if the PMF is not damped by a MHD (or other) process and therefore stays frozen into the plasma.
}

Widrow et al. 2012, for reviews) is of particular importance for cosmology. Because of the larger scale of the resulting seed magnetic field and the nature of the QCD bubble walls during a first order QCDPT, it is more likely that the QCDPT rather than the EWPT produces a PMF that accounts for the observed galactic and cluster MFs.

In this paper we consider one of several possible mechanisms of PMF generation. In particular we re-address the model proposed by Kisslinger (2003), in which the PMF is generated via QCD bubble collisions. We consider the coupling of this initial PMF with the QCD plasma, and study the dynamics during the expansion of the Universe. The main parameters of the described model are given by the QCDPT temperature $T_{\star}=0.15 \mathrm{GeV}$ and the number of relativistic degrees of freedom $g_{\star}=15$. The interactions between the PMF and the QCD plasma is studied through numerical MHD simulations using the PENCIL CoDE (see http://pencil-code.googlecode.com/). We discuss observational signatures of such a QCDPT PMF, including observed MFs in galaxies and clusters. We employ natural units with $\hbar=1=c$ and gaussian units for the MHD formulation.

The outline of the paper is as follows: In Section 2 we describe the PMF generation model. In Section 3 we determine the spatial and temporal characteristics of the generated PMF. The results of our analysis, including the dynamics of the PMF, are presented in Section 4, where we discuss the resulting MF in galaxies and clusters. Conclusions are presented in Section 5.

\section{MAGNETIC FIELD GENERATION MODEL}

In contrast to the EWPT, the QCDPT involves the treatment of QCD, which, unlike the electroweak theory, is non-perturbative. Therefore a valid theory starting from basic QCD theory, rather than a model, must be 
able to treat non-perturbative QCD. In Kisslinger (2003), instantons form gluonic bubble walls and it is the interior gluonic wall that leads to the magnetic seed described below satisfy that criterion. In this early work the main interest was the prediction of polarization correlations in cosmic microwave background radiation (CMBR). As we can see below the magnitude of the resulting MF is too small for current CMBR observations, but it might be measured in the future. Because this scenario starts from basic non-perturbative QCD theory and successfully predicts a primary magnetic field which has the overall properties that are promising for the PMF, we use it in our present work.

In this section we briefly describe the PMF scenario proposed by Kisslinger (2003). In Section 2.1 we review the magnetic field and helicity density created during the QCDPT, and in Section 2.2 we give values of these quantities at the present time. Recent lattice QCD studies have shown that the QCDPT is first order, so bubbles form and collide (see De Risi et al. 2008; Fraga \& Misher 2009; Bessa et al. 2009; Schwarz \& Stuke 2009; Schettler et al. 2011; Boeckel \& Schaffner-Bielich 2011, and references therein); additional references are given by Kahniashvili et al. (2010b). The first order QCDPT can result in the generation of a MF through two (or more) bubble collisions.

\subsection{Magnetic Field and Helicity During the QCDPT}

The QCD phase transition critical temperature is defined as $T_{\star} \simeq 0.15 \mathrm{GeV}$. A gluonic wall is created as two bubbles collide, and a magnetic wall is formed by the interaction of the nucleons with the gluonic wall. The electromagnetic interaction Lagrangian is

$$
\mathcal{L}^{\mathrm{int}}=-e \bar{\Psi} \gamma^{\mu} A_{\mu}^{\mathrm{em}} \Psi
$$

where $\Psi$ is the nucleon field operator, $A^{\mathrm{em}}$ is the electromagnetic 4-potential, and $\gamma^{\mu}$ are the Dirac matrices. In Kisslinger (2003) it was shown that the interaction of the quarks in the nucleons with the gluonic wall align the nucleon magnetic dipole moments, producing a $B$ field orthogonal to the gluonic wall.

Using an instanton model for the gluonic wall oriented in the $x-y$ direction (say), one obtains for $B_{z}(\boldsymbol{x})$ at the time of the QCDPT, with $T=T_{\star}$,

$$
B_{z}(\boldsymbol{x})=B_{\star}^{(\mathrm{QCD})} e^{-b^{2}\left(x^{2}+y^{2}\right)} e^{-M_{n}^{2} z^{2}},
$$

where $b^{-1}=d_{H} \simeq$ a few $\mathrm{km}=$ horizon size at the end of the QCDPT $\left(t \simeq 10^{-4} \mathrm{~s}\right)$ and $M_{n}^{-1}=0.2 \mathrm{fm}$. $B_{\star}^{\mathrm{QCD}}$, the magnitude of the MF within the wall of thickness $\zeta$, is (see Kisslinger 2003)

$$
B_{\star}^{(\mathrm{QCD})} \simeq \frac{1}{\zeta \Lambda_{\mathrm{QCD}}} \frac{e}{2 M_{n}} \times\left\langle\bar{\Psi} \sigma_{21} \gamma_{5} \Psi\right\rangle,
$$

where $\Lambda_{\mathrm{QCD}} \simeq 0.15 \mathrm{GeV}$ is the $\mathrm{QCD}$ momentum scale, $\gamma_{5}=i \gamma^{0} \gamma^{1} \gamma^{2} \gamma^{3}$, and $\sigma_{21}=i \gamma_{2} \gamma_{1}=i \gamma^{2} \gamma^{1}$. A similar form had been derived earlier using the domain wall model of Forbes \& Zhitnitsky (2000). The value for $B^{(\mathrm{QCD})}$ was found to be

$$
\begin{aligned}
B_{\star}^{(\mathrm{QCD})} \simeq 0.39 \frac{e}{\pi} \Lambda_{\mathrm{QCD}}^{2} & \simeq 1.5 \times 10^{-3} \mathrm{GeV}^{2} \\
& \simeq 2.2 \times 10^{16} \mathrm{G} .
\end{aligned}
$$

The asterisk indicates that we refer to the initial value of the MF at the time of the QCDPT.

We now discuss the magnetic helicity created during the QCDPT using the scenario proposed by Kisslinger (2003). Magnetic helicity is an important characteristic that strongly influences the PMF dynamics. Magnetic helicity is a conserved quantity during the subsequent evolution past the QCDPT. This leads to an inverse cascade producing magnetic fields at progressively larger scales. For this to work, it is important to know the magnetic helicity that is produced by the QCDPT.

The magnetic helicity is defined as $\int \mathrm{d}^{3} x \mathbf{A} \cdot \boldsymbol{B}$, with $\boldsymbol{B}=\nabla \times \mathbf{A}$. In the domain wall model of Forbes \& Zhitnitsky (2000), the magnetic helicity density $\mathcal{H}_{M}$ is

$$
\mathcal{H}_{M}=\boldsymbol{A} \cdot \boldsymbol{B}=A_{z} B_{z},
$$

for a PMF in the $z$ direction, as discussed above. Because of strong $\mathrm{CP}$ violation during the QCDPT, magnetic helicity is produced through the alignment of magnetic and electric dipole moments of the nucleons. Thus, the electric field satisfies $E_{z} \simeq B_{z}$ (see Forbes \& Zhitnitsky 2000). From Maxwell's equations in the Weyl gauge we have

$$
\boldsymbol{E}=-\frac{1}{c} \frac{\partial \boldsymbol{A}}{\partial t} \quad \text { or } \quad A_{z} \simeq-E_{z} \tau,
$$

where $\tau \simeq 1 / \Lambda_{\mathrm{QCD}}$ is the timescale for the QCDPT. From Eqs. (5) and (6) one finds

$$
\begin{aligned}
\mathcal{H}_{M, \star}^{\mathrm{QCD}} & \simeq B_{z}^{2} / \Lambda_{\mathrm{QCD}} \\
& \simeq\left(0.22 \times 10^{17} \mathrm{G}\right)^{2} /(0.15 \mathrm{GeV}),
\end{aligned}
$$

where we have assumed statistical homogeneity, so the result is gauge-independent.

\subsection{Comoving Values of Magnetic Field and Helicity}

The simple dilation due to the expansion of the Universe significantly reduces the amplitude of both the MF and the magnetic helicity created during the QCDPT. Defining $a_{\star}$ and $a_{0}$ as the scale factors at the time of the QCDPT and today, respectively, we have

$$
\frac{a_{\star}}{a_{0}} \simeq 10^{-12}\left(\frac{0.15 \mathrm{GeV}}{T_{\star}}\right)\left(\frac{15}{g_{\star}}\right)^{1 / 3},
$$

with $g_{*}=15, T_{\star}=0.15 \mathrm{GeV}$.

The comoving (present) value of the PMF field $B_{\text {in }}$ (the subscript "in" indicates that the QCD field is an initial $\mathrm{PMF}$ for further developed MHD dynamics) is given by $B_{\text {in }}=\left(a_{\star} / a_{0}\right)^{2} \times B_{\star}^{(\mathrm{QCD})}$, which results in

$$
B_{\text {in }} \simeq 2 \times 10^{-8} \mathrm{G} \text {. }
$$

As in the case of the PMF amplitude, magnetic helicity density experiences dilution due to the expansion of the Universe. The comoving (initial) value of the magnetic helicity density is given by

$$
\mathcal{H}_{M, \text { in }}=\left(\frac{a_{\star}}{a_{0}}\right)^{3} \times \mathcal{H}_{M, \star}^{\mathrm{QCD}} \simeq 10^{-39}\left(\mathrm{G}^{2} \cdot \mathrm{Mpc}\right) .
$$

This value is extremely small, and it is almost $\times 10^{19}$ smaller than the maximal allowed magnetic helicity (see below). Such a small value of magnetic helicity density 
is due to the thickness of the magnetic wall; see Eq. (2) and Kisslinger (2003). On the other hand several studies indicate strong $\mathrm{CP}$ violation during QCDPT (Kharzeev 2006; Voloshin 2004; Creutz 2011). In this case magnetic helicity can reach its maximal value, if we assume the MF to be correlated over the Hubble scale $\lambda_{H_{\star}}$, as will be explained below. Being more conservative we assume that the MF correlation should coincide with the bubble size $\xi_{M}$, see Sec. 3. The resulting magnetic helicity will then be smaller than the maximal one by a factor of the order of $\xi_{M} / \lambda_{H_{\star}}$. As we will see in Sec. 4, the duration of the process is long enough to ensure that the maximal value of magnetic helicity is reached during the subsequent evolution.

\section{MAGNETIC FIELD SPECTRUM}

Following our earlier studies Kahniashvili et al. 2010a) we treat the initial PMF energy density $\mathcal{E}_{M}$ as magnetic energy density injected into the cosmological plasma at the comoving length scale $\lambda_{0}$ which corresponds to the QCD bubble size. We recall that the PMF has been generated on the thin surfaces between colliding bubbles, while the correlation length scale of this PMF might be associated with the bubble length scale. In the following, we assume that the PMF spectrum in Fourier space is sharply peaked at $k_{0}=2 \pi / \lambda_{0}$. After generation, the PMF evolution (during the $\mathrm{PT}$ ) depends sensitively on the length scale under consideration and on the presence of magnetic helicity (see Harrison 1970; Turner \& Widrow 1988; Cornwall 1997; Jackiw \& Pi 2000; Garretson et al. 1992; Field \& Carroll 2000; Giovannini 2000; Vachaspati 2001; Campanelli 2007, 2009; Durrer et al. 2011, for magnetic helicity generation mechanisms). The expansion of the Universe leads to additional effects, in particular to a faster growth of the PMF correlation length. A distinctive effect is the different time behavior of the PMF decay.

In the cosmological context most important is the difference between the growth of the comoving length scale $(L \propto a)$ and the Hubble radius $\left(H^{-1} \propto t\right.$, where $t$ is physical time). This leads to additional effects in the PMF evolution (and damping) (see Son 1999; Baneriee \& Jedamzik 2003; Caprini et al. 2009). Note that, to describe properly the dynamics of the perturbations in the expanding Universe, it is appropriate to switch to comoving quantities and to describe the processes in terms of conformal time $\eta$ (Brandenburg et al. 1996). After this procedure the MHD equations include the effects of the expansion while retaining their conventional flat spacetime form. To keep the description as simple as possible we work with dimensionless quantities, such as the normalized wavenumber ${ }^{2} \gamma=\lambda_{0} / H_{\star}^{-1}$ and normalized energy density defined below.

The coupling between the PMF and the plasma leads to a spreading of the fixed scale PMF over a wide range of length scales, thus forming the PMF spectrum. After a few turnover times the modified PMF spectrum is established (see Sec. 4 for details of the simulations).

To show the coupling between the initial PT-generated $\mathrm{MF}$ and the plasma we give here the basic MHD equa-

\footnotetext{
2 Here the subscript $\star$ indicates again the moment of the PMF generation. $\gamma$ can be associated with the number of PMF bubbles within the Hubble radius, $N \propto \gamma^{3}$. This value depends on the PT model: for the QCDPT we assume $\gamma \simeq 0.15$.
}

tions for an incompressible conducting fluid (Biskamp 2003)

$$
\begin{aligned}
& {\left[\frac{\partial}{\partial \eta}+(\boldsymbol{v} \cdot \boldsymbol{\nabla})-\nu \nabla^{2}\right] \boldsymbol{v}=(\boldsymbol{b} \cdot \boldsymbol{\nabla}) \boldsymbol{b}-\nabla p+\boldsymbol{f}_{K},} \\
& {\left[\frac{\partial}{\partial \eta}+(\boldsymbol{v} \cdot \boldsymbol{\nabla})-\lambda \nabla^{2}\right] \boldsymbol{b}=(\boldsymbol{b} \cdot \boldsymbol{\nabla}) \boldsymbol{v}+\boldsymbol{\nabla} \times \boldsymbol{f}_{M},}
\end{aligned}
$$

with $\boldsymbol{\nabla} \cdot \boldsymbol{b}=0$, where $\eta$ is the conformal time, $\boldsymbol{v}(\boldsymbol{x}, \eta)$ is the fluid velocity, $\boldsymbol{b}(\boldsymbol{x}, \eta) \equiv \boldsymbol{B}(\boldsymbol{x}, \eta) / \sqrt{4 \pi w}$ is the normalized MF, $\boldsymbol{f}_{K}(\boldsymbol{x}, \eta)$ and $\boldsymbol{f}_{M}(\boldsymbol{x}, \eta)$ are external forces driving the flow and the magnetic field $\left(\boldsymbol{f}_{K}=\boldsymbol{f}_{M}=0\right.$ for the results presented below, but $\boldsymbol{f}_{M} \neq 0$ for producing initial conditions), $\nu$ is the comoving viscosity of the fluid, $\lambda$ is the comoving resistivity, $w=\rho+p$ is the enthalpy, $\rho$ is the energy density, and $p$ is the pressure of the plasma. Here we are interested in the radiation dominated epoch.

To proceed we derive the Fourier transform of the PMF two point correlation function as

$\left\langle b_{i}^{*}(\boldsymbol{k}, \eta) b_{j}\left(\boldsymbol{k}^{\prime}, \eta+\tau\right)\right\rangle=(2 \pi)^{3} \delta\left(\boldsymbol{k}-\boldsymbol{k}^{\prime}\right) F_{i j}^{M}(\boldsymbol{k}, \tau) . f[\kappa(k), \tau]$,

Such a presentation allows a direct analogy with hydrodynamic turbulence (Landau \& Lifshitz 1987). In fact, $b_{i}$ represents the Alfvén velocity. The normalized energy density of the PMF is then $\mathcal{E}_{M}=\left\langle\boldsymbol{b}^{2}\right\rangle / 2$, while the kinetic energy density is $\mathcal{E}_{K}=\left\langle\boldsymbol{v}^{2}\right\rangle / 2$, and the spectral correlation tensor is

$$
\frac{F_{i j}^{M}(\boldsymbol{k}, \tau)}{(2 \pi)^{3}}=P_{i j}(\boldsymbol{k}) \frac{E_{M}(k, \tau)}{4 \pi k^{2}}+i \varepsilon_{i j l} k_{l} \frac{H_{M}(k, \tau)}{8 \pi k^{2}} .
$$

Here, $P_{i j}(\boldsymbol{k})=\delta_{i j}-k_{i} k_{j} / k^{2}$ is the projection operator, $\delta_{i j}$ is the Kronecker delta, $k=|\boldsymbol{k}|, \varepsilon_{i j l}$ is the totally antisymmetric tensor, and $\kappa(k)$ is an autocorrelation function that determines the characteristic function $f[\kappa(k), \tau]$ describing the temporal decorrelation of turbulent fluctuations. The function $H_{M}(k, \eta)$ is the magnetic helicity spectrum. Note that $E_{M}(k)=k^{2} P_{B}(k) / \pi^{2}$, where $P_{B}(k)$ is the MF power spectrum.

The power spectra of magnetic energy $E_{M}(k, \eta)$ and magnetic helicity $H_{M}(k, \eta)$ are related to magnetic energy density and helicity density through $\mathcal{E}_{M}(\eta)=$ $\int_{0}^{\infty} \mathrm{d} k E_{M}(k, \eta)$ and $\mathcal{H}_{M}(\eta)=\int_{0}^{\infty} \mathrm{d} k H_{M}(k, \eta)$, respectively. The magnetic correlation length,

$$
\xi_{M}(\eta)=\frac{1}{\mathcal{E}_{M}(\eta)} \int_{0}^{\infty} \mathrm{d} k k^{-1} E_{M}(k, \eta),
$$

corresponds to the largest eddy length scale. All configurations of the MF must satisfy the "realizability condition" (Biskamp 2003)

$$
\left|\mathcal{H}_{M}(\eta)\right| \leq 2 \xi_{M}(\eta) \mathcal{E}_{M}(\eta) .
$$

Also, the velocity energy density spectrum $E_{K}(k, \eta)$ is related to the kinetic energy of the turbulent motions through $\mathcal{E}_{K}(\eta)=\int_{0}^{\infty} \mathrm{d} k E_{K}(k, \eta)$.

One of the main characteristics of the PMF is the correlation length and its growth. The maximal correlation length $\xi_{\max }$ for a causally generated PMF cannot exceed the Hubble radius ${ }^{3}$ at the time of generation $H_{\star}^{-1}$. The

3 The inflation generated PMF (Turner \& Widrow 1988; Ratra 1992) correlation length can exceed the Hubble horizon today. 
comoving length corresponding to the Hubble radius at generation is inversely proportional to the temperature $T_{\star}$,

$$
\lambda_{H_{\star}}=5.3 \times 10^{-7} \mathrm{Mpc}\left(\frac{0.15 \mathrm{GeV}}{T_{\star}}\right)\left(\frac{15}{g_{\star}}\right)^{1 / 6},
$$

and is equal to $0.5 \mathrm{pc}$ for the QCDPT with $g_{\star}=15$ and $T_{\star}=0.15 \mathrm{GeV}$.

The PMF spectrum is characterized not only by its spatial distribution, but also by its characteristic times: (i) the largest-size eddy turnover time $\tau_{0} \simeq l_{0} / v_{A}$ (where $v_{A}$ is the r.m.s. Alfvén velocity), which can also be used to determine the minimal duration of the source needed to justify the use of the stationary turbulence approximation (Proudman 1952; Monin \& Yaglom 1975); (ii) the direct cascade time-scale of the turbulence $\tau_{\mathrm{dc}}$; and (iii) the large-scale turbulence decay time $\tau_{\mathrm{ls}}$.

The temporal characteristics of the MHD turbulence is given through the form of $f(\kappa(k), \tau)$, which is due to the complex process of MHD turbulence decorrelation (Terry \& Smith 2007) and is currently not fully understood. To proceed we employ Kraichnan's approach (Kraichnan 1964) and specify the decorrelation function $f_{\mathrm{dc}}\left[\kappa\left(k_{\mathrm{ph}}\right), \tau\right]=\exp \left[-\pi \kappa^{2}\left(k_{\mathrm{ph}}\right) \tau^{2} / 4\right]$ defined within the inertial range, $k_{0}<k<k_{d}$. Here $\tau$ is the duration of the turbulence process and $\kappa\left(k_{\mathrm{ph}}\right)=\bar{\varepsilon}_{M}^{1 / 3} k_{\mathrm{ph}}^{2 / 3} / \sqrt{2 \pi}$, where $k_{\mathrm{ph}}$ is the physical wavenumber related to the comoving $k$ through $k_{\mathrm{ph}}(a)=k a_{0} / a\left(a_{0}\right.$ is the value of the scale factor now), and $\bar{\varepsilon}_{M}$ is the proper dissipation rate per unit enthalpy. Hence, we have (Kahniashvili et al. 2011)

$$
f_{\mathrm{dc}}[\bar{k}, \tau]=\exp \left[-\frac{2 \pi^{2}}{9}\left(\frac{\tau}{\tau_{0}}\right)^{2} \bar{k}^{4 / 3}\right] .
$$

Here, $\bar{k}=k / k_{0}$ is the normalized wavenumber and $\tau_{0}$ corresponds to the largest eddy turnover time. It is clear that after switching off the forcing, the turbulent motions are decorrelated within a few turnover times, and are in fact irrelevant to influence the large-scale MF.

\section{GROWTH OF CORRELATION LENGTH IN HELICAL TURBULENCE}

To assess the importance of a small initial magnetic helicity, we perform direct numerical simulations of decaying MHD turbulence with an initial MF of finite relative magnetic helicity using different values, and a correlation length $\xi_{M}$ that is small compared with the scale of the domain $\lambda_{1}$.

\subsection{Simulation technique}

We solve the compressible equations with the pressure given by $p=\rho c_{s}^{2}$, where $c_{s}=1 / \sqrt{3}$ is the sound speed for an ultra-relativistic gas. Following our earlier work (Kahniashvili et al. 2010a), we solve the equations governing equations for the logarithmic density $\ln \rho$, the velocity $\boldsymbol{v}$, and the magnetic vector potential $\boldsymbol{A}$, in the form

$$
\begin{aligned}
\frac{\mathrm{D} \ln \rho}{\mathrm{D} \eta} & =-\boldsymbol{\nabla} \cdot \boldsymbol{v} \\
\frac{\mathrm{D} \boldsymbol{v}}{\mathrm{D} \eta} & =\boldsymbol{J} \times \boldsymbol{B}-c_{s}^{2} \boldsymbol{\nabla} \ln \rho+\boldsymbol{f}_{\mathrm{visc}}
\end{aligned}
$$

$$
\frac{\partial \boldsymbol{A}}{\partial \eta}=\boldsymbol{v} \times \boldsymbol{B}+\boldsymbol{f}_{M}+\lambda \nabla^{2} \boldsymbol{A},
$$

where $\mathrm{D} / \mathrm{D} \eta=\partial / \partial \eta+\boldsymbol{v} \cdot \boldsymbol{\nabla}$ is the advective derivative, $\boldsymbol{f}_{\text {visc }}=\nu\left(\nabla^{2} \boldsymbol{v}+\frac{1}{3} \boldsymbol{\nabla} \boldsymbol{\nabla} \cdot \boldsymbol{v}+\boldsymbol{G}\right)$ is the viscous force in the compressible case with constant $\nu$ and $G_{i}=$ $\mathrm{S}_{i j} \nabla_{j} \ln \rho$ as well as $\mathrm{S}_{i j}=\frac{1}{2}\left(v_{i, j}+v_{j, i}\right)-\frac{1}{3} \delta_{i j} v_{k, k}$ being the trace-free rate of strain tensor. Furthermore, $\boldsymbol{J}=\boldsymbol{\nabla} \times \boldsymbol{B} / 4 \pi$ is the normalized current density. We emphasize that $\boldsymbol{f}_{M}=\mathbf{0}$, except for producing initial conditions, as explained below.

The bulk motions are always slow enough, so compressibility effects are not important. Similar to before, we express the magnetic field in Alfvén units, but now based on the volume average enthalpy, i.e., $\boldsymbol{b} \equiv \boldsymbol{B} / \sqrt{4 \pi\langle w\rangle}$, where $w=\frac{4}{3} \rho$ for an ultrarelativistic gas. We use $512^{3}$ meshpoints in a domain of size $(2 \pi)^{3}$, so the lowest wavenumber in the domain is $k_{1}=1$. We choose $\nu=\eta=10^{-5}$ in units of $c_{s} / k_{1}$.

\subsection{Initial conditions}

A suitable initial condition is produced by simulating for a short time interval $\left(\Delta t \approx 0.5 \lambda_{1} / c_{s}\right)$ with a random $\delta$-correlated magnetic forcing term $\boldsymbol{f}_{M}$ in the evolution equation for the magnetic vector potential. This forcing term consists of plane monochromatic waves with wavenumber $k_{0}$ and fractional helicity $\left\langle\boldsymbol{f}_{M} \cdot \boldsymbol{\nabla} \times \boldsymbol{f}_{M}\right\rangle /\left\langle k_{0} \boldsymbol{f}_{M}^{2}\right\rangle=2 \sigma /\left(1+\sigma^{2}\right)$; in the following we quote the value of $\sigma$. This procedure has the advantage that the magnetic and velocity fields used then for the subsequent decay calculations are obtained from a self-consistent solution to the MHD equations.

\subsection{Growth of helical structures}

In Figure 1 we show spectra of magnetic and kinetic energy, as well as the magnetic helicity scaled by $k / 2$, for a run with $\sigma=0.03$. Initially, $k H_{\mathrm{M}}(k, \eta) / 2$ is well below the value of $E_{M}(k, \eta)$. However, at later times the two approach each other at large scales. This shows that the relative magnetic helicity increases during the decay. For the four times shown in Figure1, the rms Mach number, $v_{\mathrm{rms}} / c_{s}$, is $0.05,0.025,0.012$, and $0.007 ; b_{\mathrm{rms}} / v_{\mathrm{rms}}$ is around 3.4, and the Reynolds numbers $v_{\mathrm{rms}} \xi_{M} / \nu$ are roughly 270 all cases.

The growth of turbulent structures is particularly clear in the magnetic field (Figure 2). The magnetic field drives correspondingly larger scale structures also in the velocity field. However, there are also strong small-scale fluctuations in the velocity field that are not visible in the magnetic field; see the second row of Figure 2 .

In agreement with earlier simulations, we find that at small scales the magnetic energy is re-distributed by a direct cascade with a Kolmogorov-type spectrum, $E_{M}(k) \propto k^{-5 / 3}$. At large scales a Batchelor spectrum ${ }^{4}$, $E_{M}(k) \propto k^{4}$, is established, which was used as initial condition already in Brandenburg et al. (1996). This spectrum is in agreement with the analytical description of Durrer \& Caprini (2003) who derived this result from the requirement of causality and the divergence free condition. The earlier study of Hogan (1983), which thus violates causality for magnetic energy, yielded a white noise

\footnotetext{
4 Sometimes this spectral distribution, $E_{M}(k) \propto k^{4}$, is called a
} von Kármán spectrum (Pope 2000). 

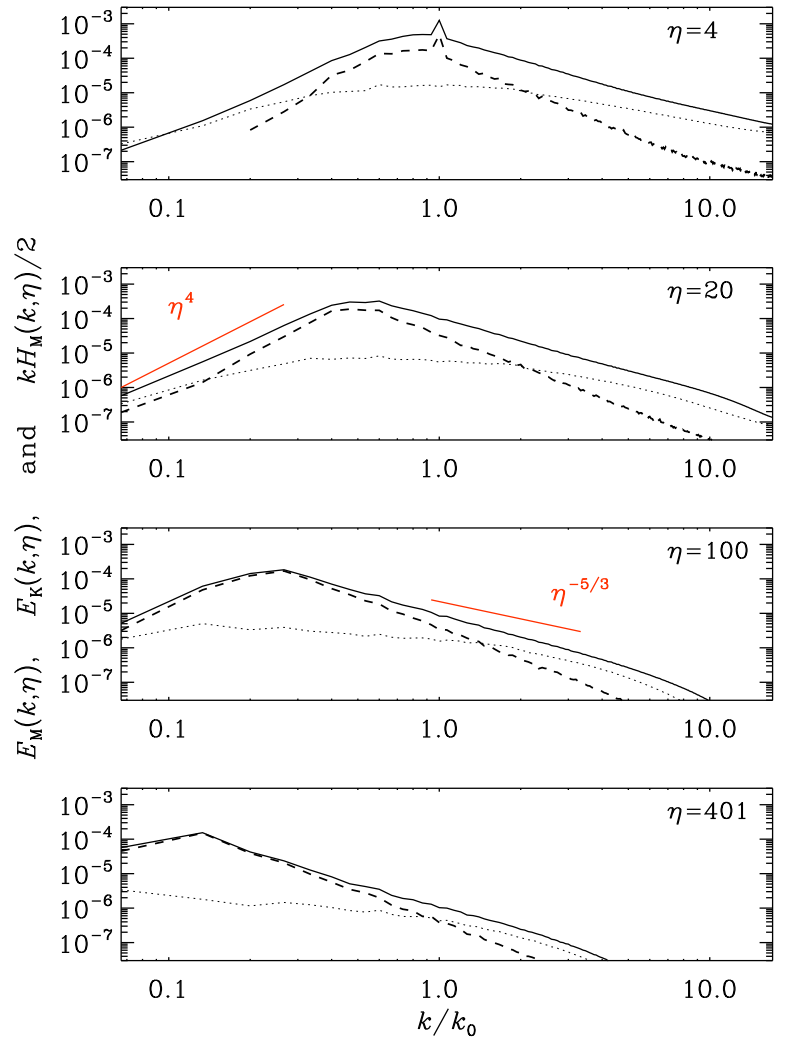

FIG. 1. - Spectra of magnetic energy (solid lines), kinetic energy (dotted), and magnetic helicity scaled by $k / 2$ (dashed) for a run with $\sigma=0.03$ at three different times. At early times, $H_{M}(k, \eta)$ can be negative at small values of $k$, which explains why the dashed line terminates in those cases.

spectrum $E(k) \propto k^{2}$ (Saffman spectrum) which we do observe for the spectral distribution of the kinetic energy $E_{K}(k) \propto k^{2}$.

\subsection{Growth of turbulent length scales}

The evolution of magnetic correlation length and magnetic energy during the MHD turbulence decay can be described using two indices $n_{\xi}$ and $n_{E}$ :

$$
\begin{aligned}
& \xi_{M}(\eta)=\xi_{M}\left(\eta_{0}\right)\left(\frac{\eta}{\eta_{0}}\right)^{n_{\xi}}, \\
& \mathcal{E}_{M}(\eta)=\mathcal{E}_{M}\left(\eta_{0}\right)\left(\frac{\eta}{\eta_{0}}\right)^{n_{E}} .
\end{aligned}
$$

In this case, we can model the spectral energy density of the PMF using time-dependent large- and small-scale ranges:

$$
E_{M}(k, \eta)=E_{0}(\eta)\left\{\begin{array}{ll}
\bar{k}^{4} & \text { when } k<k_{I}(\eta) \\
\bar{k}^{-5 / 3} & \text { when } k>k_{I}(\eta)
\end{array},\right.
$$

where $\bar{k}=k / k_{I}$ and $k_{I}(\eta)=2 \pi / \xi_{M}(\eta)$. Hence, the evolution of the spectral amplitude $E_{0}$ for a given magnetic field spectrum will be [see Equations (22) and (23)]:

$$
E_{0}(\eta)=\frac{5}{17 \pi} \xi_{M}\left(\eta_{0}\right) \mathcal{E}_{M}\left(\eta_{0}\right)\left(\frac{\eta}{\eta_{0}}\right)^{n_{\xi}+n_{E}} .
$$

Magnetic helicity crucially affects the evolution of the PMF (Biskamp \& Müller 1999, 2000; Son 1999;
Christensson et al. 2001, 2005; Banerjee \& Jedamzik 2003, 2004; Campanelli 2007). If the PMF has been generated with small magnetic helicity, there are two main stages during the development of the MF spectrum: during the first stage (sometimes called direct cascade) the PMF dynamics is similar to that of the non-helical MF. The energy cascades from large to small scales where it decorrelates and dissipates: this is a standard forward cascade development. Since magnetic helicity is conserved, its fractional value increases and thus the end of this first stage is characterized by releasing turbulence to a maximally helical state (Banerjee \& Jedamzik 2003; Christensson et al. 2005) when the realizability condition (16) is reached, the inverse-cascade stage starts. The conservation of magnetic helicity implies that the magnetic energy density decays in inverse proportion to the correlation length growth during the inverse cascade. The realizability condition implies that

$$
\xi_{M}(\eta) \geq \xi_{M}^{\min }(\eta) \equiv\left|\mathcal{H}_{M}(\eta)\right| / 2 \mathcal{E}_{M}(\eta),
$$

so there is a minimum value for the correlation length. In Figure 3 we plot $\xi_{M}(\eta)$ and $\xi_{M}^{\min }(\eta)$ for $\sigma=1,0.1$, and 0.03. It turns out that, especially in the latter case with $\sigma=0.03$, the increase of $\xi_{M}$ remains slow $\left(\sim \eta^{1 / 2}\right)$ as long as $\xi_{M}(\eta) \gg \xi_{M}^{\min }(\eta)$. However, since $\mathcal{H}_{M}$ is essentially constant and $\mathcal{E}_{M}$ decreases approximately like $\eta^{-1}$, the value of $\xi_{M}^{\min }(\eta)$ soon reaches $\xi_{M}(\eta)$. When that happens, the field is essentially fully helical and the correlation length and the magnetic energy density evolve according to $\xi_{M} \sim \eta^{2 / 3}$ and $\mathcal{E}_{M} \sim \eta^{-2 / 3}$, respectively. Hence, we recover two distinctive phases in the MHD turbulence decay process: evolution of a weakly helical turbulence with $n_{\xi}=1 / 2$ and $n_{E}=-1$, and fully helical turbulence with $n_{\xi}=2 / 3$ and $n_{E}=-2 / 3$. Note that in the latter case $E_{0}(\eta) \propto \xi_{M}(\eta) \mathcal{E}_{M}(\eta)=$ const [see Eq. (25)] and the inverse cascade develops. Our results are in excellent agreement with Biskamp \& Müller (1999), Biskamp \& Müller (2000), Baneriee \& Jedamzik (2003), and Campanelli (2007). The dynamical process of PMF coupling with the cosmic plasma stops at the moment of recombination after which the PMF develops more slowly (Brandenburg et al. 1997).

To calculate the time $\eta_{\text {fully }}$ when a fully helical state is reached, we only need to know the initial values $\xi_{M}\left(\eta_{0}\right)$ and $\xi_{M}^{\min }\left(\eta_{0}\right)$. Since the latter approaches the former like $\eta^{1 / 2}$, the result is $\eta_{\text {fully }}=\eta_{0}\left[\xi_{M}\left(\eta_{0}\right) / \xi_{M}^{\min }\left(\eta_{0}\right)\right]^{2}$. Thus, in terms of the initial values of $\mathcal{E}_{M}$ and $\mathcal{H}_{M}$, a fully helical state is reached at the time

$$
\eta_{\text {fully }}=4 \eta_{0} \xi_{M}^{2} \mathcal{E}_{M}^{2} / \mathcal{H}_{M}^{2}
$$

Note that this time increases quadratically with decreasing initial value of $\mathcal{H}_{M}$. In case of the strong $\mathrm{CP}$ violation during the QCDPT, when the initial magnetic helicity can reach values that are only $\xi_{M} / \lambda_{H_{\star}}$ times less than the maximal one (see Sec. 2), we get $\eta_{\text {fully }}=\eta_{0} / \gamma^{2}$.

\subsection{Observed Magnetic Fields}

Galactic and cluster MFs are usually measured through Faraday rotation (see Vallée 2004) and, as mentioned above, the value of the coherent magnetic field is of the order of a few $\mu \mathrm{G}$ with a typical coherence scale of 

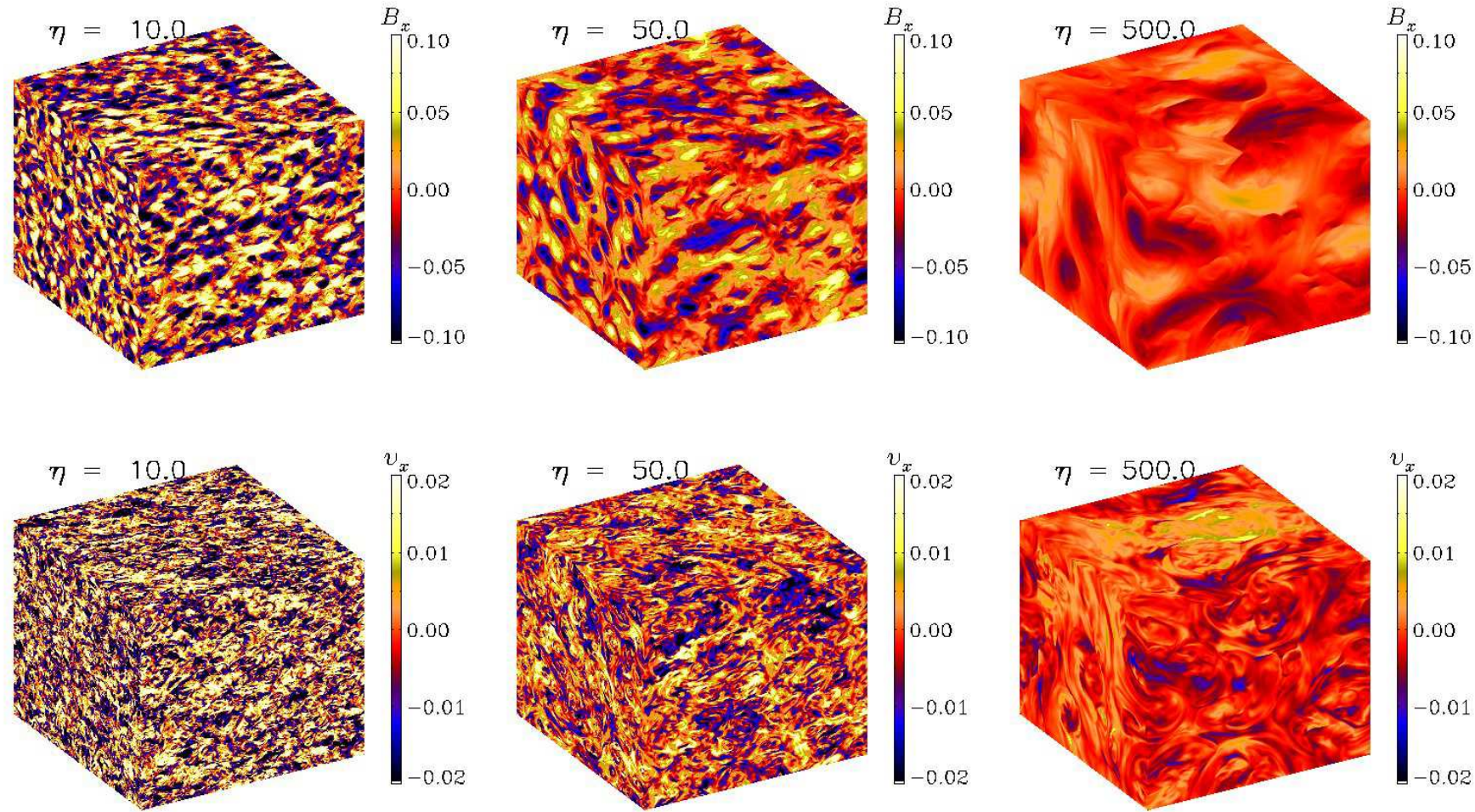

Fig. 2.- Visualizations of $B_{x}$ (upper row) and $v_{x}$ (lower row) at three times during the magnetic decay of a weakly helical field with $\sigma=0.03$.

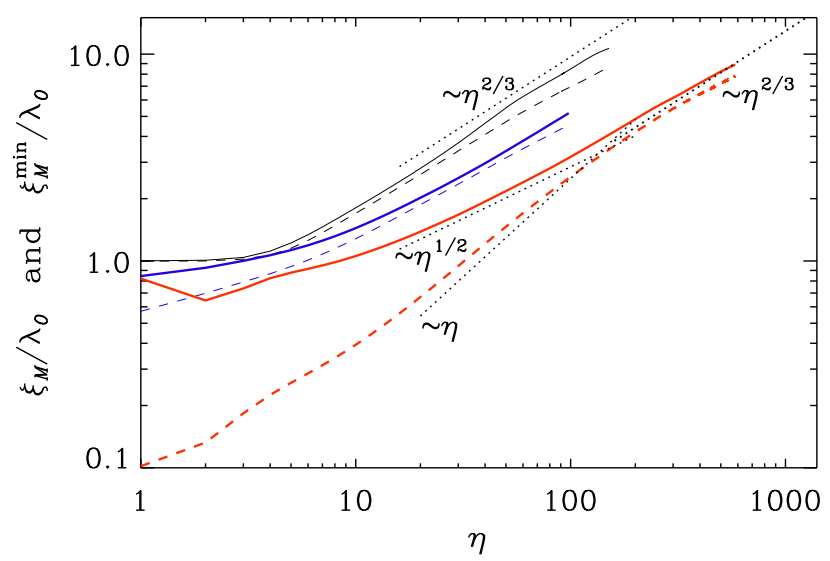

Fig. 3. - Evolution of $\xi_{M}(\eta)$ (solid) and $\xi_{M}^{\min }(\eta)$ (dashed) for $\sigma=1$ (black), 0.1 (blue) and 0.03 (red).

$10 \mathrm{kpc},{ }^{5}$ and cluster MFs have lower limits of the order of $10^{-6} \mathrm{G}$, and at least a few $\mathrm{nG}$, with similar coherence scales (Clarke et al. 2001) and additional lower limits on the steepness of the magnetic power spectrum in clusters. Furthermore, simulations starting from a constant comoving magnetic fields of $10^{-11} \mathrm{G}$ suggest that magnetic field generation in clusters can be sufficiently strong to explain Faraday rotation measurements (Dolag et al. 2002; Jedamzik \& Sigl 2011).

${ }^{5}$ Strong MFs have been detected through Faraday rotation of distant quasars proving that the MFs comparable to those observed today are seen at high redshift $z \sim 3$ (Bernet et al 2008).

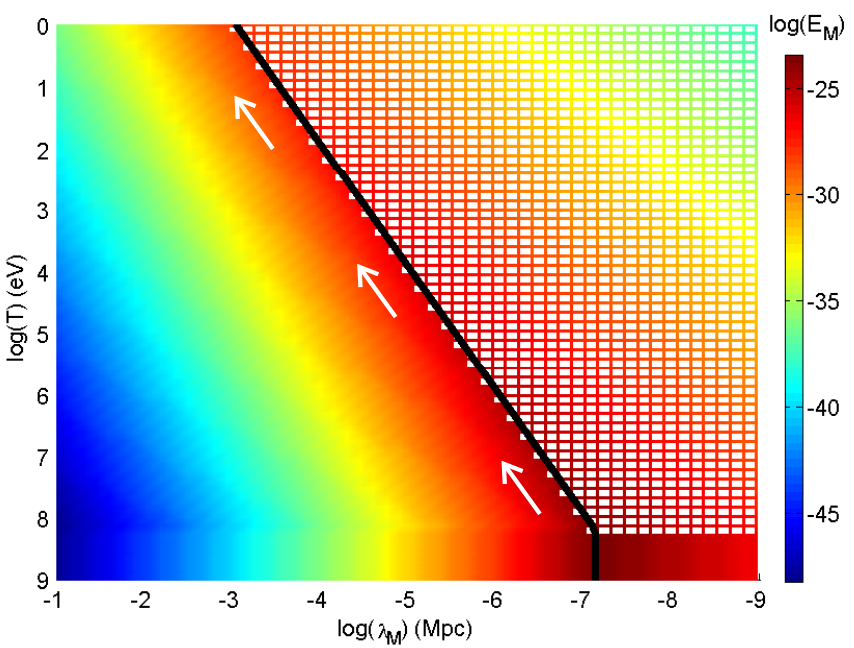

FIG. 4.- Spectral energy density of the turbulent magnetic field $\log E_{M}(k)$ (color coded) in a representation of magnetic correlation length versus temperature. The thick solid line shows the evolution of the magnetic correlation length $\xi_{M}(T)$. The magnetic correlation length starts to grow after the QCD phase transition at $T_{\star}=0.15 \mathrm{GeV}$, when $\xi_{M}=0.075 \mathrm{pc}$. The transparent dashed area corresponds to decorrelated magnetic field. White arrows show the direction of the evolution during the expansion of the Universe. Here $n_{\xi}=-1 / 2$ and $n_{E}=1$.

Figure 4 shows the spectral energy density of the QCDPT-generated MF (see Kisslinger 2003) with respect to temperature and correlation length in weakly helical turbulence. Initially the integral scale of the MHD turbulence is set by the QCDPT bubble scale (lower right 


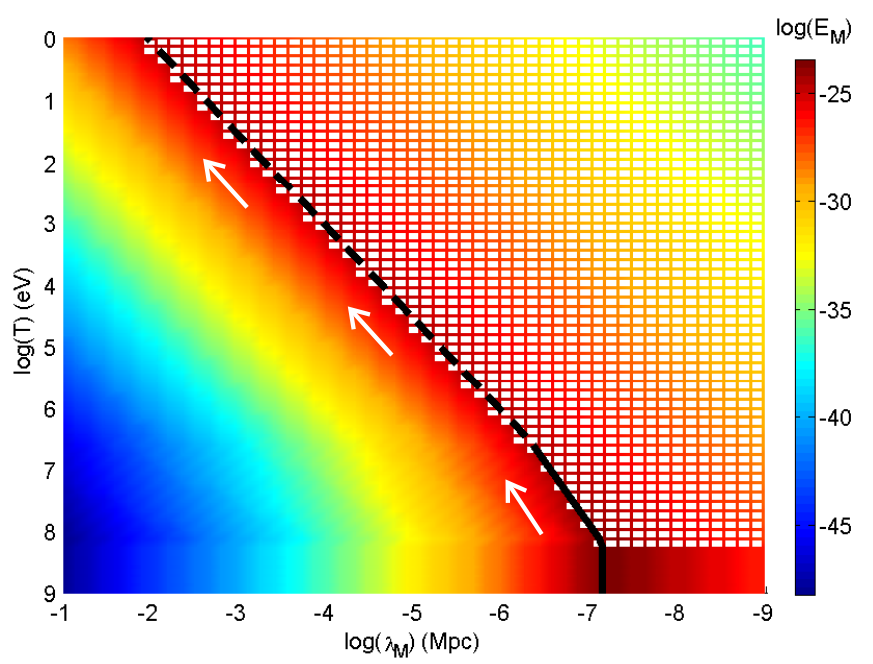

FIG. 5.- Similar to Fig. 4 but for the case in which the magnetic field reaches a fully helical state within the considered expansion time of the Universe. Initially, during the growth of magnetic helicity correlation length (solid line) and energy evolve according to $n_{\xi}=-1 / 2$ and $n_{E}=1$. After reaching a fully helical state, correlation length (dashed line) and energy evolve according to $n_{\xi}=-2 / 3$ and $n_{E}=2 / 3$.

corner of the diagram). The thick solid line marks the division between the evolution of large-scale (plain colored region) and small-scale (hashed colored region) magnetic fields. White arrows indicate the direction of the evolution during the Universe expansion. At scales below the integral scale of the turbulence, the magnetic field undergoes exponential decorrelation; see Eq. (18). The integral scale of the MHD turbulence increases, reaching $\xi_{M}=1 \mathrm{kpc}$ at $T=1 \mathrm{eV}$. Here we have used $n_{\xi}=1 / 2$, $n_{E}=-1$, with initial magnetic helicity corresponding to that given by Eq. (10).

Figure 5 shows the spectral energy density of a QCDPT-generated MF in the case when the initial helical turbulence reaches the fully helical case during the expansion of the Universe with $\eta_{\text {fully }} / \eta_{0}=1 /(0.15)^{2}$. The thick solid line marks the evolution of the magnetic correlation length until the magnetic helicity reaches its maximally allowed value. In this time interval the decay law for weakly helical turbulence with $n_{\xi}=1 / 2$ and $n_{E}=-1$ is applied. After the time $\eta_{\text {fully }}$ when maximal magnetic helicity is reached, the correlation length follows the black dashed line and the MF evolution follows that of the fully helical case with $n_{\xi}=2 / 3$ and $n_{E}=-2 / 3$. The integral scale of the MHD turbulence reaches $\xi_{M}=10 \mathrm{kpc}$ at $T=1 \mathrm{eV}$.

The presented model is somewhat idealized since it ignores the time of Silk damping due to large correlation lengths for photon and neutrino viscosity (see Jedamzik \& Sigl 2011). This is justified since it only delays the evolution but does not destroy the field (Brandenburg et al. 1997). Therefore, we can present here only upper values for QCDPT MFs within the model by Kisslinger (2003).

The final amplitude of the MF can be estimated through two different approaches. (i) We compute the total magnetic energy density, i.e. $\mathcal{E}_{M}=\int_{0}^{\infty} \mathrm{d} k E_{M}(k)$ and make the assumption that all energy is again given only at one scale that corresponds to the integral scale at this moment, i.e. $B_{\text {eff }}=\sqrt{8 \pi \mathcal{E}_{M}}$. (ii) Another approach is to compute the strength of the MF, $B(\lambda)$, at a given scale $\lambda$. Since observations (Vallée 2004) do not allow us to properly reconstruct the configuration of the MF we adopt first an "effective" MF approach (see Kahniashvili et al. 2011). The resulting value of the effective MF in our model of weakly helical turbulence with $\xi_{M}=1 \mathrm{kpc}$ reaches $5 \times 10^{-4} \mathrm{nG}$, while in the case of a fully helical configuration with $\xi_{M}=10 \mathrm{kpc}$ we find $7 \times 10^{-3} \mathrm{nG}$.

\section{CONCLUSIONS}

In this paper we have considered QCDPT-generated PMFs and their evolution in an expanding Universe accounting for the effects of MHD turbulence to explain the seed MFs of clusters and galaxies. We consider the MF generation model proposed by Kisslinger (2003), which yields an initial state of weakly helical MHD turbulence. We also study the possibility of strong $\mathrm{CP}$ violation according to Forbes \& Zhitnitsky (2000), which yields an initial state with much higher magnetic helicity at a time when maximal helicity of the MHD turbulence is reached during the expansion of the Universe. The initial seed MF is generated via QCDPT bubble collisions with a comoving correlation length of the order of $0.1 \mathrm{pc}$ and with a comoving amplitude of the order of $20 \mathrm{nG}$. The initial magnetic helicity is determined by the thickness of the surface between two colliding bubbles and is extremely small if no strong CP violation is assumed (Kharzeev 2006; Voloshin 2004; Creutz 2011). During the expansion of the Universe there are different processes that affect the correlation length and the strength of the MF: first of all, during the PT the field is initially peaked at a given scale and then spreads out within a wide range of wavelengths, establishing a Kolmogorov-like spectrum, $E_{M}(k) \propto k^{-5 / 3}$, at small scales and a Batchelor spectrum, $E_{M}(k) \propto k^{4}$, at large scales. If the PMF was generated without being maximally helical, the magnetic helicity experiences a steady growth. One of the results obtained in this paper is an estimate of the timescale within which the field starts to be fully helical. In the case of an extremely weakly helical field (Kisslinger 2003), the available time to produce a fully helical PMF may be too long. The growth of the correlation length follows then a $\xi_{M} \propto T^{-1 / 2}$ law. For moderate or reasonably small initial magnetic helicity (even for $\sigma \geq 10^{-6}-10^{-5}$ ), the evolution timescale is long enough so that during the first stage of evolution, magnetic helicity grows to its maximal value. During the next stages (after magnetic helicity has reached its maximal value) the correlation length experiences a steady growth with the scaling law $\xi_{M} \propto T^{-2 / 3}$ while the energy density is decreasing in the opposite way keeping magnetic helicity almost constant. Finally, at recombination the growth of the correlation length slows down. The resulting correlation length in the most optimistic scenarios is around $10 \mathrm{kpc}$ and the amplitude of the MF is around $0.007 \mathrm{nG}$. Assuming that the MF is amplified during the growth of structures (Dolag et al. 2002), such a field might well be strong enough to explain the observed MF in galaxies and clusters. On the other hand, observations of the CMB fluctuations are sensitive to PMFs of the order of a few $\mathrm{nG}$ (see Shaw \& Lewis 2010; Yamazaki et al. 2010, and references therein). 
Another possible signature of QCDPT-generated magnetic fields is a gravitational wave signal (Kahniashvili et al. 2010b) that might be indirectly detected through pulsar timing (Durrer et al. 2011). The gravitational waves signal from PTs is usually computed assuming short duration of the source (either turbulence or PMF anisotropic stress). On the other hand, due to the free decay of MHD turbulence, the source of gravitational waves acts also after the end of PTs. For short duration sources, the peak frequency of the gravitational waves is fully determined by the source characteristics. In particular, for QCDPT-generated gravitational waves it is far too weak to be detected though gravitational waves via ground or space based missions. Long duration sources might in principle substantially change the peak frequency as well as the amplitude of the signal. We plan to address this issue in future work.
We appreciate helpful comments and discussions with L. Campanelli, C. Caprini, R. Durrer, A. Kosowsky, K, Kunze, A. Neronov, B. Ratra, and T. Vachaspati. We acknowledge partial support from Computing resources have been provided by the Swedish National Allocations Committee at the Center for Parallel Computers at the Royal Institute of Technology in Stockholm and by Carnegie Mellon University supercomputer center. We acknowledge partial support from Swiss National Science Foundation SCOPES grant no. 128040, NSF grant AST1109180 and NASA Astrophysics Theory Program grant NNXlOAC85G. This work was supported in part by the European Research Council under the AstroDyn Research Project 227952 and the Swedish Research Council grant 621-2007-4064. T.K. acknowledges the ICTP associate membership program. A.B. and A.T. acknowledge the McWilliams Center for Cosmology for hospitality.

\section{REFERENCES}

Banerjee, R., \& Jedamzik, K. 2004, Phys. Rev. D, 70, 123003

Banerjee, R., \& Jedamzik, K. 2003, Phys. Rev. Lett., 91, 251301

Beck, R., Brandenburg, A., Moss, D., Shukurov, A., \& Sokoloff, D. 1996, ARA\&A, 34, 155

Bernet, M. L., Miniati, F., Lilly, S. J., Kronberg, P. P., \& Dessauges-Zavadsky, M. 2008, Nature, 454, 302.

Clarke, T. E., Kronberg, P. P., \& Böhringer, H. 2001, ApJ, 547, L111

Bessa, A., Fraga, F. S. \& Mintz, B. W. 2009, Phys. Rev. D, 79, 034012

Biskamp, D. 2003, Magnetohydrodynamic Turbulence (Cambridge University, Cambridge)

Biskamp, D., \& Müller, W.-C. 2000, Phys. Plasma, 7, 4889

Biskamp, D., \& Müller, W.-C. 1999, Phys. Rev. Lett., 83, 2195

Boeckel, T., \& Schaffner-Bielich, J. 2011, Phys. Rev. D, 85, 103506

Brandenburg, A., Enqvist, K., \& Olesen, P. 1996, Phys. Rev. D, 54,1291

Campanelli, L. 2009, Int. J. Modern Phys. D, 18, 1395

Campanelli, L. 2007, Phys. Rev. Lett., 98, 251302

Caprini, C., Durrer, R., \& Fenu, E. 2009, JCAP, 0911, 001

Caprini, C., Durrer, R., \& Siemens, X. 2010, Phys. Rev. D, 82, 063511

Christensson, M., Hindmarsh, M., \& Brandenburg, A. 2005, Astron. Nachr., 326, 393

Christensson, M., Hindmarsh, M., \& Brandenburg, A. 2001, Phys. Rev. E, 70, 056405

Cornwall, J. 1997, Phys. Rev. D, 56, 6146

Creutz, M., 2011, Phys. Rev. D 83, 016005.

De Risi, G., Harko, T., Lobo, F. S. N., \& Pun, C. S. J. 2008, Nucl. Phys. B, 805, 190

Dermer, C. D., Cavaldini, M., Razzaque, S., Finke, J.D., Chiang, J., \& Lott, B. 2011, ApJ, 733, L21

Brandenburg, A., Enqvist, K., \& Olesen, P. 1997, Phys. Lett. B., 392, 395

Dolag, K., Bartelmann, M., \& Lesch, H. 2002, A\&A, 387, 383

Dolag, K., Kachelriess, M., Ostapchenko, S., \& Tomas, R. 2011, ApJ, 727, L4

Durrer, R., \& Caprini, C. 2003, JCAP, 0311, 010

Durrer, R., Hollenstein, L., \& Jain, R. K. 2011, JCAP, 1103, 037

Field, G.B. \& Carroll, S. M. 2000, Phys. Rev. D62, 103008.

Forbes, M., \& Zhitnitsky, A. 2000, Phys. Rev. Lett., 85, 5268

Fraga, E. S., \& Mizher, A. J. 2009, Nucl. Phys. A, 820, 103C

W. D. Garretson, W. D., Field, G. B. \& Carroll, S. M. 1992, Phys. Rev. D, 46, 5346

Giovannini, M. 2000, Phys. Rev. D, 61, 063004
Grasso D., \& Rubinstein, H. R. 2001, Phys. Rev. D, 348, 163

Harrison, E. R. 1970, MNRAS, 147, 279

Hogan, C. J. 1983, Phys. Rev. Lett., 51, 1488

Huan, H., Weisgarber, T., Arlen, T., \& Wakely, S. P. 2011, ApJ, $735, \mathrm{~L} 28$

Jackiw, R., \& Pi, S. Y. 2000, Phys. Rev. D, 61, 105015

Jedamzik, K., \& Sigl, G. 2011, Phys. Rev. D, 83, 103005

Kahniashvili, T., Brandenburg, A., Tevzadze, A. G., \& Ratra, B. 2010a, Phys. Rev. D, 81, 123002

Kahniashvili, T., Kisslinger, L., \& Stevens, T. 2010b,

Phys. Rev. D, 81, 023004

Kahniashvili, T., Tevzadze, A. G., \& Ratra, B., 2011 ApJ, 726, 78

Kharzeev, D., 2006, Phys. Lett. B 633, 260.

Kisslinger, L. S. 2003, Phys. Rev. D, 043516

Kraichnan, R. H. 1964, Phys. Fluids, 7, 1163

Landau, L. D., \& Lifshitz, E. M., Fluid Mechanics, Pergamon Press, NY.

Monin, A. S., \& Yaglom, A. M. 1975, Statistical Fluid Mechanics (MIT Press, Cambridge, MA)

Neronov, A., \& Vovk, I. 2010, Science 328, 73

Pope, B. P. 2000, Turbulent Flows (Cambridge University,

Cambridge).

Proudman, I., 1952, Proc. R. Soc. London A, 214, 119

Ratra, B., 1992, ApJ, 391, L1

Schettler, S. Boeckel, T., \& Schaffner-Bielich, J. 2011, Prog. Part. Nucl. Phys., 66, 266

Shaw, J. R., \& A. Lewis, A. 2010, arXiv:1006.4242 [astro-ph.CO]

Son, D. T. 1999, Phys. Rev. D, 59, 063008

Schwarz, D. J., \& Stuke, M. 2009, JCAP, 0911, 025

Takahashi, K., Mori, M., Ichiki, K., \& Inoue, S. 2012, ApJ, 744, L7

Tavecchio, F., Ghisellini, G., Foschini, L., Bonnoli, G., Ghirlanda, G., \& Coppi,P. 2010, MNRAS, 406, L70

Taylor, A. M., Vovk, I., \& Neronov, A. 2011, A\&A, 529, A144

Terry, W. P., \& Smith, K. W. 2007, ApJ, 665, 402

Turner, M. S., \& Widrow, L. M. 1988, Phys. Rev. D, 37, 2743

Vachaspati, T. 2001, Phys. Rev. Lett., 87, 251302

Vallée, J. P. 2004, New A Rev., 48, 763

Voloshin, S. A., 2004, Phys. Rev. C 70, 057901

Yamazaki, D. G., Ichiki, K,, Kajino, T., \& Mathews, G. J. 2012, Adv. Astron., 2010, 586590

Widrow, L. M. 2002, Rev. Mod. Phys., 74, 775

Widrow, L. M., Ryu, D., Schleicher, D., Subramanian, K., Tsagas, C. G., \& Treumann, R. A. 2012, Spa. Sci. Rev., 166, 37 